\title{
Simplified measurements of the humidity coefficient of torque transducers in calibration laboratories
}

\author{
Andreas Brüge \\ Physikalisch-Technische Bundesanstalt, Bundesallee 100, 38116 Braunschweig, Germany
}

ABSTRACT

Several simple methods to test the humidity influence on the sensitivity of torque transducers are examined. Their suitability for use in calibration laboratories and important influences are discussed. The equivalence of former determinations of the humidity effect with extensive methods to the simplified methods is demonstrated.

\section{Section: RESEARCH PAPER}

Keywords: Torque; transducer; sensitivity; humidity; measurement

Citation: Andreas Brüge, Simplified measurements of the humidity coefficient of torque transducers in calibration laboratories, Acta IMEKO, vol. 3, no. 2, article 9, June 2014, identifier: IMEKO-ACTA-03 (2014)-02-09

Editor: Paolo Carbone, University of Perugia

Received February $15^{\text {th }}, 2013$; In final form June $25^{\text {th }}, 2013$; Published June 2014

Copyright: @ 2014 IMEKO. This is an open-access article distributed under the terms of the Creative Commons Attribution 3.0 License, which permits unrestricted use, distribution, and reproduction in any medium, provided the original author and source are credited

Funding: (none reported)

Corresponding author: Andreas Brüge, e-mail: andreas.bruege@ptb.de

\section{INTRODUCTION}

Torque transducers are known to be influenced by humidity [1], [2]. Due to different levels of sealing on the surface of the sensing elements of the transducers, the deviation of the sensitivity caused by a change of humidity can vary in amount and time constants. The usual methods to measure the effect are complicated and extensive. Nevertheless, this information is essential to complement the measurement uncertainty budget of torque calibration. In calibration laboratories only few resources are available for such investigations. In order to enable these laboratories to test their transfer torque transducers at low cost, this paper describes simple methods to determine the humidity effect and demonstrates their equivalence to the extensive methods.

In section 2 a theoretical description of the effect is given. In section 3 different possibilities to alter the humidity of torque transducers are discussed, followed by a description of how the humidity coefficient could be determined. Here important sources of uncertainty are also analyzed. In section 5 the results of measurements on typical transducers are presented and in section 6 a summary is given.

\section{HUMIDITY EFFECT}

The influence of humidity is supposed to be due to the hygroscopic behaviour of the strain gauges and the adhesive layer which couples them to the elastic element. The change of their mechanical properties can affect the sensitivity of the strain gauge bridges as well as the time constants of their response, and a change in additional strain gauges for temperature compensation can cause a signal offset.

A stepwise humidity change will not act instantaneously on the bridge but in an exponential way. The effective time behaviour of the humidity $F$ in the bridge can be described as

$$
\begin{aligned}
& F(t)=F_{0}+A_{\mathrm{F}}\left(1-e^{\left(-\frac{t}{\tau_{\mathrm{F}}}\right)}\right) \\
& \text { with } t: \text { time } \\
& F_{0} \text { : initial amount of humidity } \\
& A_{\mathrm{F}} \text { : } \quad \text { change of the humidity } \\
& \tau_{\mathrm{F}}: \quad \text { time constant of the humidity. }
\end{aligned}
$$

This causes time behaviour of the transducer sensitivity $S$ as follows:

$$
\begin{aligned}
& S(t)=S_{0}+S(F)=S_{0}+A_{S}\left(1-e^{\left(-\frac{t}{\tau_{S}}\right)}\right) \\
& \text { with } t: \text { time } \\
& S_{0} \text { : initial amount of sensitivity } \\
& A_{\mathrm{S}} \text { : } \quad \text { change of the sensitivity } \\
& \tau_{\mathrm{S}} \text { : time constant of the sensitivity. }
\end{aligned}
$$


Sensitivity in this paper is the signal of the transducer at nominal value in $\mathrm{mV} / \mathrm{V}$ as is usual in the field of torque measurement.

If the alteration of humidity does not penetrate all the elements of the transducer's sensing element in the same velocity, transient effects on the sensitivity should be taken into account:

$$
\begin{aligned}
& T(t)=-A_{\mathrm{T}}\left(e^{\left(-\frac{t}{\tau_{\mathrm{T}}}\right)}\right) \\
& \text { with } T \text { : } \\
& t \\
& A_{\mathrm{T}}: \quad \text { change of the transient signal } \\
& \tau_{\mathrm{T}}: \quad \text { time constant of the transient } \\
& \text { effect. }
\end{aligned}
$$

A simulation of these functions shows the principal behaviour of them (Figure 1).

A separation of the contributions $S$ and $T$ to the total response is only possible if the initial sensitivity - valid just before the humidity step starts - of the transducer is known. The effects which affect this sensitivity depend on the method of applying the change of humidity and therefore are to be considered when a method should be recommended.

The relative coefficient of the humidity effect on the transducer sensitivity $c_{\mathrm{h}}$ can then be defined as:

$$
c_{\mathrm{h}}=\frac{S\left(t_{2}\right)-S\left(t_{1}\right)}{\bar{S}\left(t_{2}, t_{1}\right)\left(F\left(t_{2}\right)\right)-\left(F\left(t_{1}\right)\right)}
$$

A negative coefficient thus stands for an increasing sensitivity at decreasing humidity and vice versa. The unit of the relative humidity coefficient is $1 / \%_{\mathrm{rH}}$, which means "per percent point of relative humidity". In this work the value of the relative humidity is given in percent $(\%)$, and the value of a span of different levels of relative humidity as percent points of relative humidity $\left(\% \%_{\mathrm{rH}}\right)$.

\section{VARIATION OF HUMIDITY}

\subsection{Humidification of the laboratory}

The straightforward way to change humidity for the determination of the relative humidity coefficient $c_{\mathrm{h}}$ is by using an air-conditioned laboratory room with a torque calibration facility inside. Here a reference torque calibration facility is not suitable, for the humidity effects of the reference torque

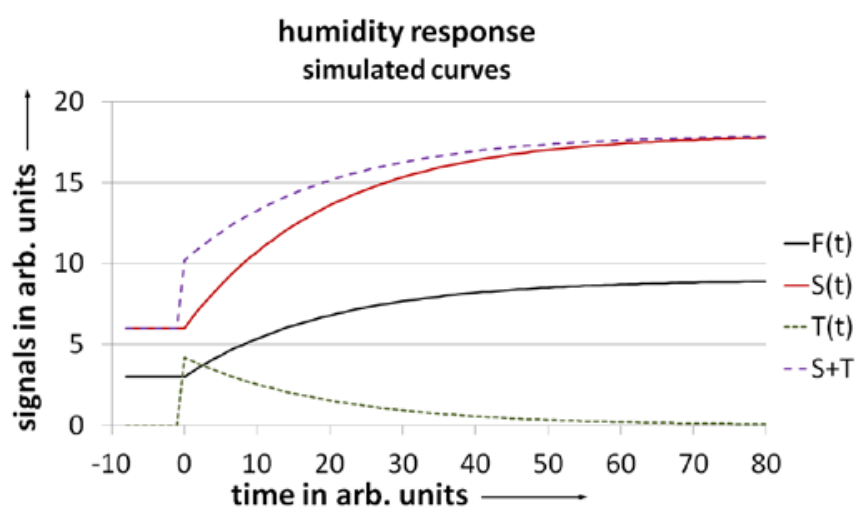

Figure 1. Simulation of the principal time behaviour of the humidity effect. transducer and of the transducer under test would combine. Therefore, a deadweight facility is necessary which is assumed to be independent of humidity. In this setup the entire room can be altered to different levels of humidity at a constant temperature.

Because of the high volume of an entire room, the alterations need a long time until stability is reached. For the same reason the stability of the humidity level is of high quality. The first observation of the humidity effect happened in this way, during a key comparison in laboratories of different humidity levels [1].

If there are more calibration facilities in the room, their results may be influenced by the humidity alteration too. Therefore, other facilities might be stopped during the humidification, so the costs of the room humidification can be much higher than economically orientated laboratories can bear.

\subsection{In-situ humidification in a chamber}

Another possibility is to construct an air-conditioned chamber around the transducer under test mounted inside the calibration facility (Figure 2).

In such a chamber, the temperature and humidity of the air should be controllable in a stable or transient way as desired. It should provide low parasitic effects due to a mechanical bypass, the behaviour of the control unit, air flow and evaporationcooling. Altogether the method is connected with high mechanical and electrical effort. When these difficulties have been overcome, the method potentially provides measurements at very stable humidity levels and with low uncertainty even in reference torque calibration facilities. Some systematic investigations of the humidity effect were performed at the Physikalisch-Technische Bundesanstalt (PTB) with such a

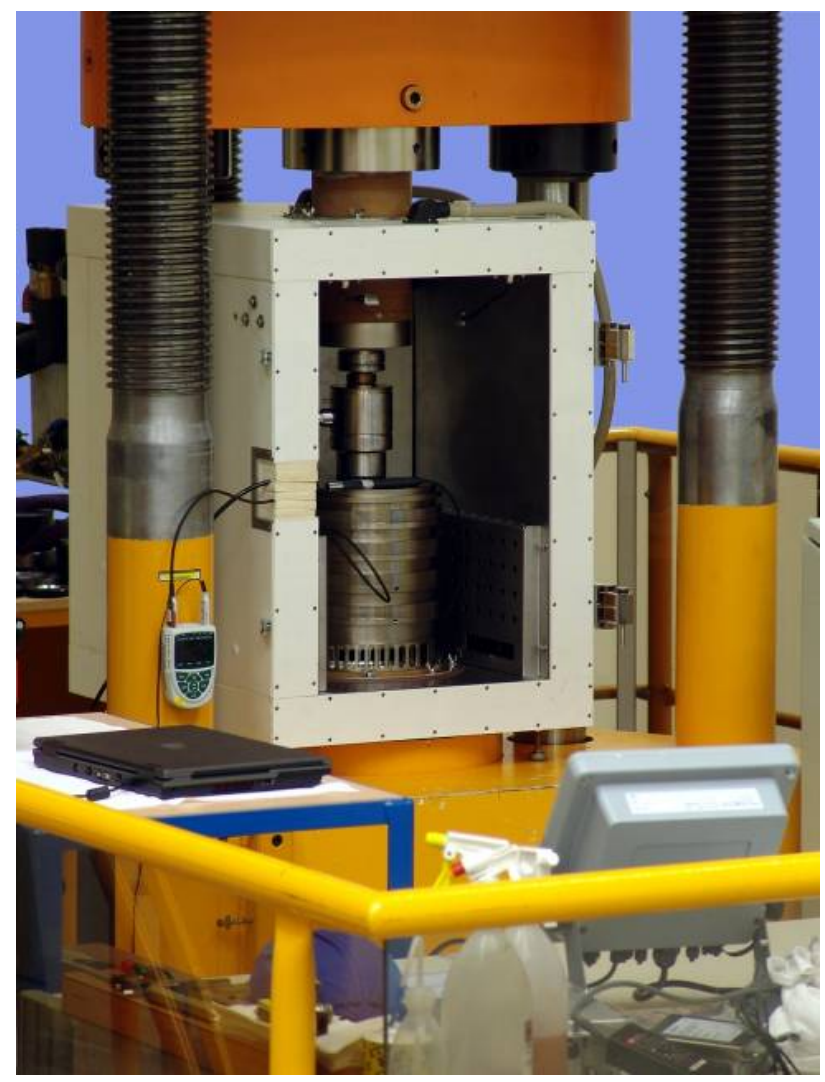

Figure 2. Example of an air-conditioned chamber integrated into a setup of a force calibration facility. 


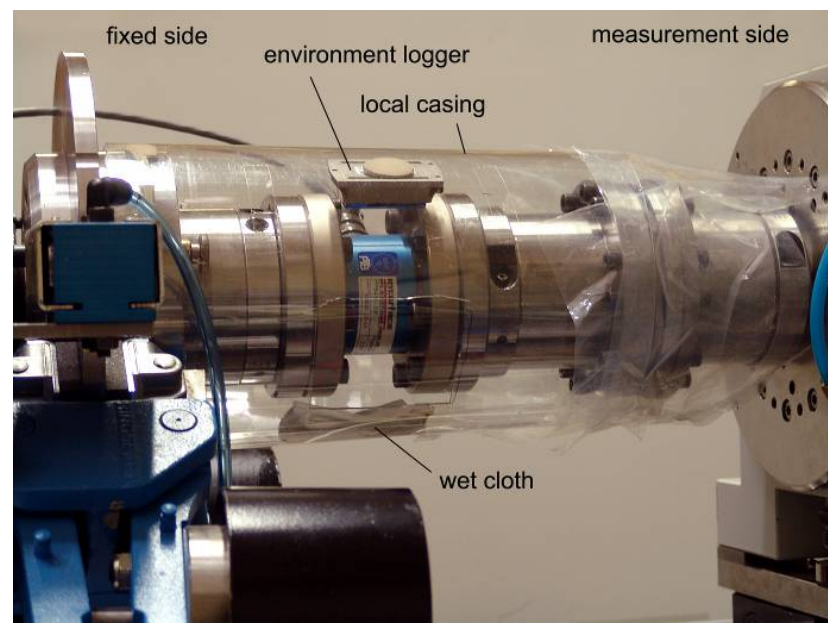

Figure 3. In-situ humidification of a torque transducer inside a provisional local casing. An environment logger is used for the determination of the development of humidity and temperature.

chamber [2] and serve as a reference for the simplified methods presented in this work.

\subsection{In-situ humidification, local casing}

The preceding methods are possible for most National Metrological Institutes (NMIs) but, for calibration laboratories, usually the effort is too high. A very simple and cheap version of a climate chamber used in this work is a casing of light acrylic (Figure 3).

Inside the casing a wet cloth or a pad of silica gel produces a relative humidity of about $80 \%$ and $20 \%$ respectively, which is measured by a small environment logger placed within the casing near the transducer. The actual level of humidity depends on the relation between the water transmission capability of the cloth or the pad, respectively, and the air leakage of the casing. In order to get higher humidity steps and to ensure small spatial humidity gradients within the casing, the leakage should be minimized. Since the casing spans both shaft ends of the transducer twisting up to several angular degrees while loaded to nominal torque, an effective seal is associated with a mechanical bypass between these shaft ends. Additionally, the casing should feature a sealed cable feedthrough.

Together with the relative humidity of the laboratory being about $45 \%$ this in-situ humidification is able to generate three levels of humidity steps. Because these levels are uncontrolled, a systematic investigation of the humidity effect with arbitrary humidity steps is not possible.

Nevertheless, the method is useful because it reaches humidity spans of more than $50 \% \mathrm{o}_{\mathrm{rH}}$, whereas the ex-situ humidification described later allows up to $35 \% \%_{\mathrm{rH}}$ only.

Additionally, this method reliably includes the transient contribution $T$ mentioned above, which is not the case for the ex-situ humidification. The possibility to observe both the initial and the final humidity level in a stable state is essential to overcome the transient effects.

\subsection{Ex-situ humidification}

Often an external climate chamber is easier to construct than a version integrated in the calibration facility or alternatively a climate cabinet may be available in the laboratory. Then the transducer under test can be humidified externally to a stable level, while the temperature stays constant at the laboratory value (Figure 4).

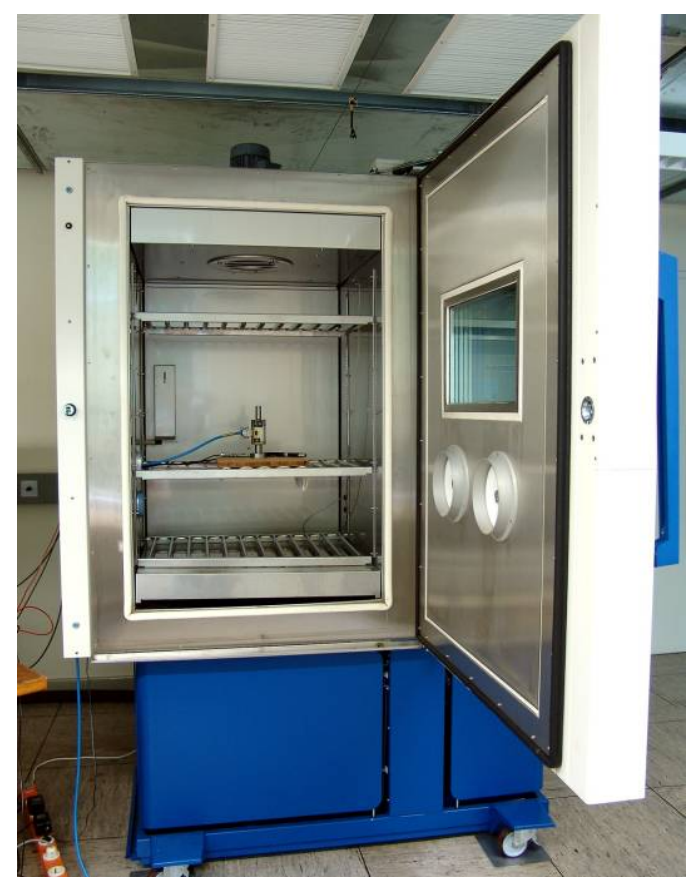

Figure 4. Ex-situ humidification of a torque transducer in a climate cabinet.

After a fast transit into the calibration facility, the measurement of the humidity effect takes place during the relaxation of the humidity impact within the sensing element to the laboratory level of humidity (Figure 5).

Because of the measurement delay due to the relocation, an extrapolation of the sensitivity response towards the origin is necessary. The initial sensitivity cannot be measured directly in this procedure.

Despite this difficulty, the use of a climate cabinet is appropriate for systematic investigations using a great number of different humidity levels. Another advantage of the ex-situ humidification is the possibility to use the calibration facility for current work while the transducer under test stays in the climate cabinet. Due to long time constants of the humidity effect this can save several days of machine time.

Additionally, for testing torque wrenches, an in-situ humidification is nearly impossible, as a local casing would induce high mechanical bypass via the lever of the wrench. Combining the simplicity of a light casing with the ex-situ method which avoids a mechanical bypass, torque wrenches can be humidified in a bag together with a wet cloth or silica gel, respectively, and an environment logger (Figure 6). Torque wrenches are usually mounted with square drives, so the

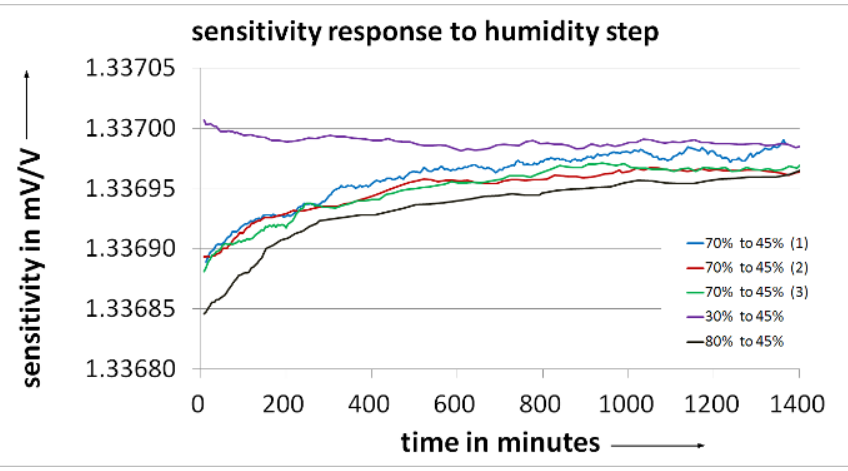

Figure 5. Response of transducer sensitivity at nominal load to different humidity deviations from laboratory level which are generated in a climate cabinet. 


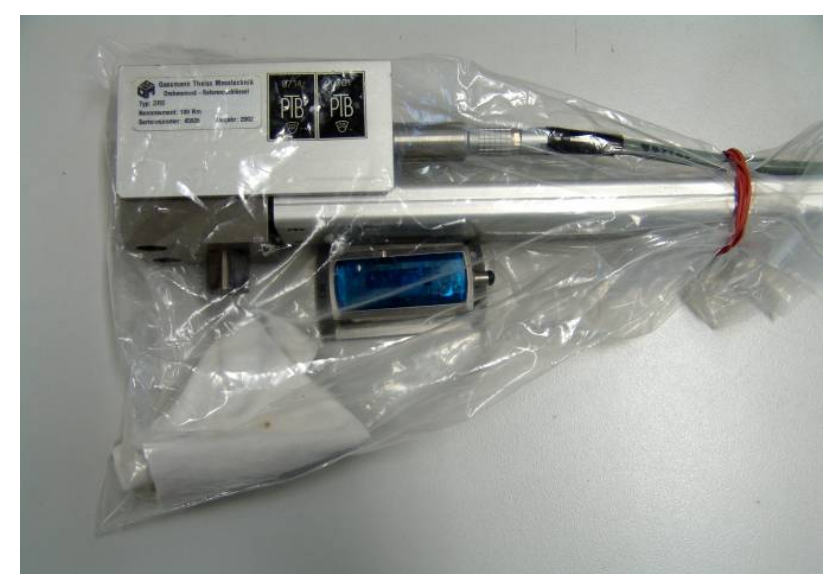

Figure 6. Ex-situ humidification of a torque wrench in a bag.

downtime of the measurement of the humidity effect is much shorter for them than for torque transducers.

A variation of the in-situ method can help for transducers which cannot be transported to and mounted into the calibration facility safely and fast enough. Here an undisturbed measurement of the initial state in laboratory ambient air without a casing is followed by a humidification using a temporary local casing. At the end of the humidification the casing is removed quickly and the relaxation to the initial sensitivity level can be observed free of mechanical bypass effects and transient effects. Here the challenge is in the construction of a casing which can be removed fast enough to minimize the downtime.

\section{MEASUREMENT OF THE HUMIDITY COEFFICIENT}

\subsection{Measurement of sensitivity}

The determination of the humidity coefficient calls for the measurement of the sensitivity of a torque transducer. Two methods lend themselves to the implementation of these measurements in a reference torque calibration facility.

Firstly, with reference calibration facilities continuously loading and unloading delivers a characteristic curve of the transducer under test by interpolation methods [4]. In this way a sensitivity measurement with good averaging is possible within one minute. Together with a preloading and a consecutive creep time, a sampling time of 5 minutes could be achieved. In addition, some important parameters of the characteristic curve are included: zero point, nonlinearity, hysteresis and return to zero.

Secondly, a stepwise loading and unloading is possible with reference calibration facilities or with deadweight facilities. Usually only three steps at $0 \%, 50 \%$ and $100 \%$ of nominal load are applied to get both a fast measurement and a value of the hysteresis. To achieve a measurement stability like that obtained with the continuous method, it is necessary to average data during each load step. This leads to a sampling time of about 10 minutes.

The first method is advantageous in investigations about the time behaviour of the humidity effect, where a fast measurement is crucial and the second method can help for measurements at transducers with high creep.

As a third method a loading with a simple manually handled lever and a deadweight was tested but rejected. A tempting idea was to work with a steady torque generated by a fixed weight while modifying the humidity at the transducer under test measurement of sensitivity

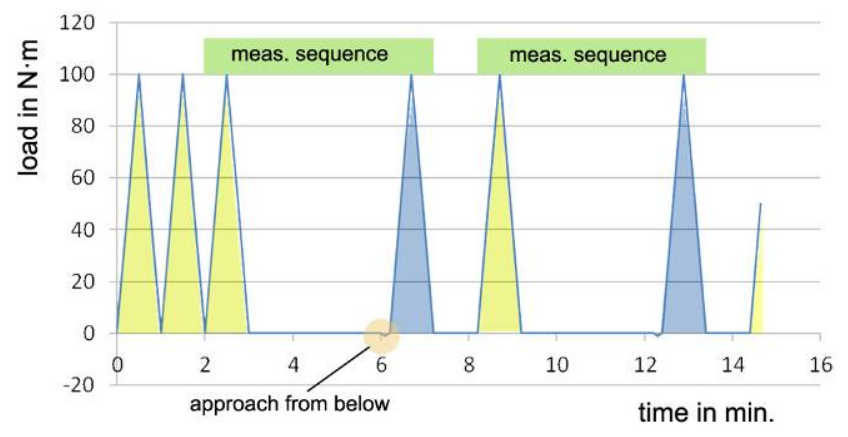

Figure 7. Achievement of the transducer sensitivity using the continuous calibration method. Each measurement sequence consists of at least one preloading (yellow), a measurement loading (blue) and a three minute break between them.

within a local casing. Then the response of the sensitivity would be available directly as the output signal of the transducer. However, because the signal wanted is superimposed by the zero signal which responds at least by the same amount to the humidity as the sensitivity itself, this method is not recommended.

In this work, measurements of the sensitivity took place with continuous loading up to the nominal torque of the transducer under test, for clockwise torque only and in one mounting position only, which was reproduced carefully for all measurements compared. Also to obtain a good comparability of conditions, all measurements were preceded by a preload up to nominal torque and a waiting time of 3 minutes (Figure 7).

After the waiting time, the load should pass through a value below the chosen virtual zero level in order to avoid hysteresis effects. Performing a sequence of measurements with a maximum rate of six measurements per minute, the time behaviour of the humidity effect can be investigated with a good time resolution in comparison to the observed time constants of the effect.

\subsection{Transient signal contributions}

As mentioned in section 2, transient effects can occur during the change of humidity level. Reactions of the local temperature due to humidity change are also possible. Regardless of the reasons, an effect depending on the change of humidity is expected to generate a signal contribution like the function $T$ in Figure 1, and in the sum an exponential function with an abrupt linear beginning could result (' $S+T$ ' in Figure 1). In the measurements these curves are often found both at exsitu and in-situ humidification at the moment of a fast change in humidity.

In contrast to effects of mechanical bypass the transient effect does not call for compensation. After the relaxation of the effect, the signal level is unchanged. Thus, in the examination it is essential to regard equilibrium states only (Figure 8). An analysis of the curve with the help of an exponential fitting function should take into account its linear start.

\subsection{Mechanical bypass}

As described in section 3.3 a well-sealed local casing breeds a mechanical bypass in the torque sensing transducer. The amount of torque deviation depends on the extent of friction of the gliding parts and on the stiffness of the deformed parts of 
sensitivity response to in-situ humidity step

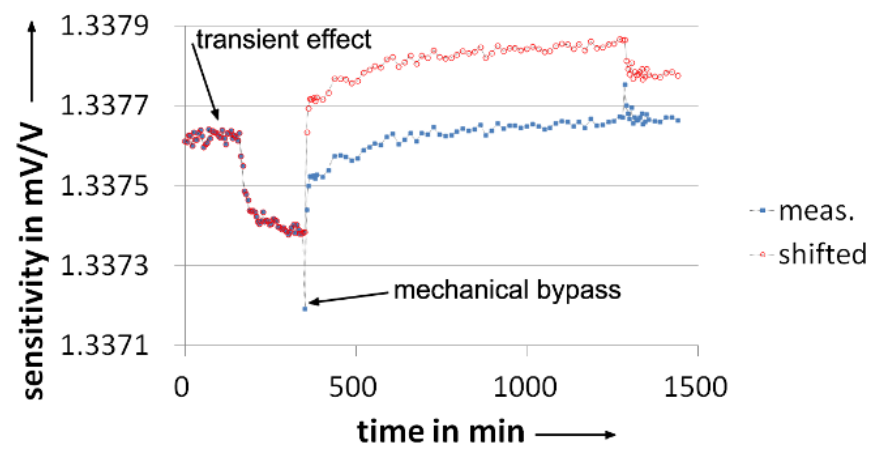

Figure 8. Sensitivity response of a transducer treated with humidity steps inside a local casing (blue dots). At the first step a pure transient effect occurs. The following two steps are influenced by the additional impact of friction due to a mechanical bypass in the casing. By appropriate compensation of the friction, the measurement can be corrected (red dots).

the casing. Consequently, the casing consists of a stiff cylinder of acrylic at the fixed end of the transducer and an overlapping blanket of flexible and gliding thin film at the measurement side. The acrylic cylinder is intended for carrying the mass of the environment logger and the wet cloth, or respectively, the gel pad. Nevertheless, changing the mass load of the cylinder and manipulating its access panel may change the position relative to the blanket and thus cause a deviation in the mechanical bypass. For the casing used in this work, the effect reaches $0.015 \mathrm{~N} \cdot \mathrm{m}$. So for a $1000 \mathrm{~N} \cdot \mathrm{m}$ transducer, this contribution can be neglected, but for transducers of smaller ranges it can be considerable. The total amount of friction in the setup could be higher, but with an untouched casing, constant friction will not affect the run of the sensitivity curve. Only additional friction due to manipulation at the casing appears in the measurements and has to be compensated for by appropriate shifting of the data points concerned (Figure 8). Although compensation is possible, it makes for additional uncertainty. Therefore, careful handling should be performed to avoid any mechanical bypass which demands a well-trained operator.

\subsection{Zero signa}

A torque transducer exposed to a changing humidity level not only shows a response in the sensitivity but also in the zero signal. The reaction of the zero signal often dominates the sensitivity by a factor of more than 10 (Figure 9).

The determination of the sensitivity includes a taring of the transducer signal at a certain load with the zero signal achieved at the beginning of the loading process. Therefore, not the entire amount of zero signal deviation affects the sensitivity measurement. Only the deviation of the zero signal taking place during the loading process cannot be compensated by the taring and has an impact on the sensitivity measurement.

The typical duration time for the continuous loading method is 30 seconds. With the stepwise loading, durations of 5 minutes were implemented. Assuming a constant change of the zero signal in short times, the contribution of the zero signal change can be derived from the time behaviour and an approximative correction of the sensitivity is possible (Figure 10).

\section{RESULTS}

zero signal influence, ex-situ humidification

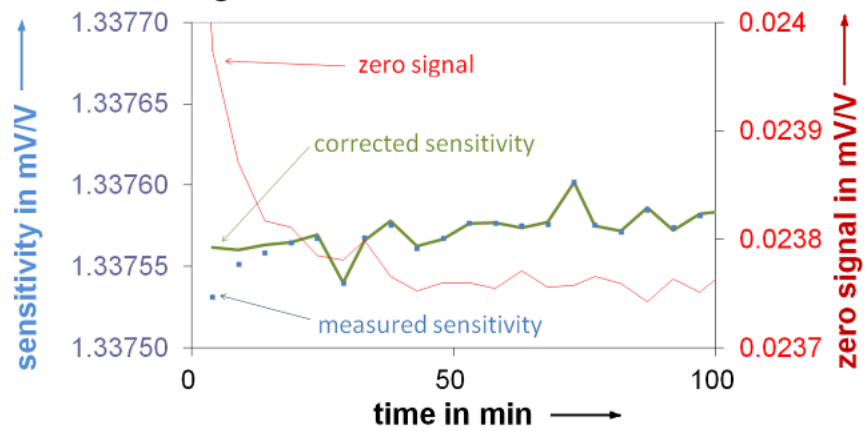

Figure 9. Sensitivity response of a transducer experiencing a relaxation of a humidity step generated in a climate cabinet (blue dots). The zero signals are gained at the beginning of each corresponding torque loading sequence, which is necessary for the sensitivity measurement (red line). A correction of the influence of the variable zero signal delivers a sensitivity curve with a flatter beginning (green line).

Values of $\iota_{\mathrm{h}}$ obtained for several state-of-the-art transducers with different nominal values are found to be about $5 \cdot 10^{-6} / \% \%_{\mathrm{rH}}$. This is close to the results of [3] where $-4 \cdot 10^{-6} / \%_{\mathrm{rH}}$ was found by changing the humidity using a chamber mounted into the calibration facility described in section 3.2.

The effects described in this work cause uncertainties of the $c_{\mathrm{h}}$ measurements of about $3 \cdot 10^{-6} / \%_{\mathrm{rH}}$. A dominating contribution is the uncertainty of the determination of the sensitivity.

At ex-situ humidification the downtime during relocation requires an extrapolation of the sensitivity curve, so here the change of the sensitivity at the first two measurements indicates the uncertainty of the sensitivity.

At in-situ setups the noise of the sensitivity measurements and the doubtful compensation of mechanical bypass comes to the fore. As $c_{\mathrm{h}}$ is defined as relative to humidity steps, the relative uncertainty increases for decreasing humidity steps. Because humidity steps above $40 \% \%_{\mathrm{rH}}$ are not accessible relative to the ambient humidity of the laboratory, a sequence of a wet cloth and a drying gel pad is necessary. With this advance the influence of the mechanical bypass is increased because the casing has to stay permanently and there is no measurement possible between stable states without the casing.

The uncertainty of the humidity step measurement is estimated at about $\pm 2 \%{ }_{\mathrm{rH}}$. This is possible with the environment logger, because only differential measurements are

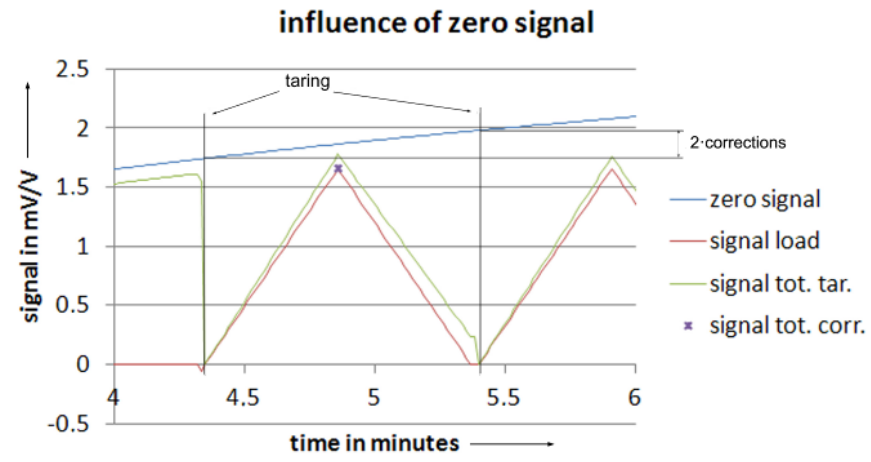

Figure 10. Influence of a changing zero signal on a measurement tared at the beginning of the loading. The contribution of the zero signal to the total signal can be estimated by a linear approximation of half of the zero signal increase during the time between two tarings. Thereby, a corrected value of the full load signal (cross) can be calculated. 


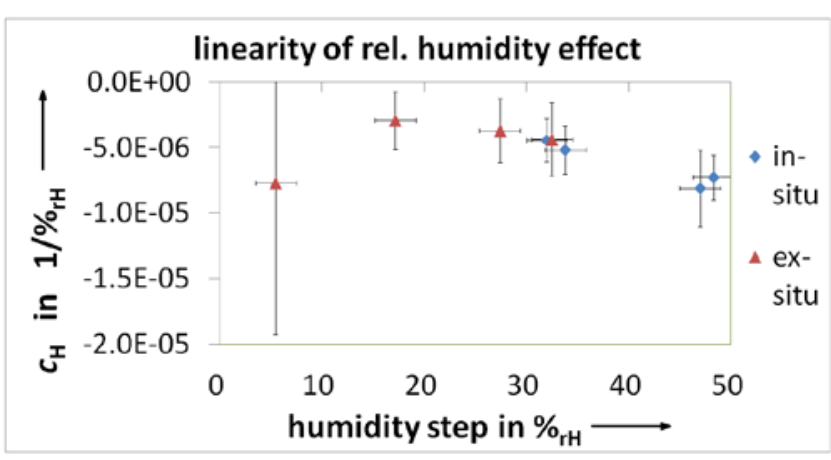

Figure 11. Values of $c_{\mathrm{h}}$ obtained with different humidification methods at a typical transfer torque transducer $(100 \mathrm{~N} \cdot \mathrm{m})$ demonstrating the equivalence of the methods. The run at different humidity steps suggests the nonlinear behaviour of $c_{\mathrm{h}}$. The error bars are calculated from the uncertainty of the measurements of respectively the humidity steps and the transducer sensitivity (see main text).

taken. The short-term change in temperature due to cooling effects by the humidity amounts to $0.1 \mathrm{~K}$. With a typical relative temperature coefficient of torque transducers of $1 \cdot 10^{-6} / \mathrm{K}$, this effect can be neglected here.

Considering these limitations, measurements at a typical transfer torque transducer yield values of $c_{\mathrm{h}}$ which are similar to former measurements [3] and are consistent for both methods of humidification used in this work (Figure 11).

The measurements at different humidity steps in consideration of the measurement uncertainty allow an interpretation both as linear and as nonlinear behaviour of $c_{\mathrm{h}}$. Examining the absolute signal response to the humidity steps in spite of a relative response, the nonlinear character of the measurements becomes more evident (Figure 12).

Because of blending measurements during increasing and decreasing humidity and because of performing humidity steps between humidity levels below and above laboratory equilibrium it is impossible to mark a reference point. Nevertheless, the data points in Figure 12 seem to be consistent in spite of the lack of a well defined working point.

In the daily work of a laboratory humidity alterations of less than $20 \%{ }_{\mathrm{rH}}$ are usual. In this range a possible nonlinearity of $c_{\mathrm{h}}$ is negligible. For the determination of $c_{\mathrm{h}}$ with the methods described in this paper, humidity steps of more than $30 \%{ }_{\mathrm{rH}}$ are opportune. Thereby, the values of $c_{\mathrm{h}}$ are tending towards overestimation which is not harmful for the calculation of a robust uncertainty budget.

\section{SUMMARY}

The simplified humidity tests described in this paper can deliver estimations of $c_{\mathrm{h}}$ with uncertainties of $3 \cdot 10^{-6} / \%_{\mathrm{rH}}$ which are small compared to the needs of calibration laboratories, where often providing information is sufficient if the coefficient's range is $10^{-6} / \%_{\mathrm{rH}}$ or $10^{-5} / \%_{\mathrm{rH}}$. With the help of these tests, the requirements for climate specifications of the air conditioning in the laboratories and for the transportation and storage conditions of transducers can be established properly.

The results verify the equivalence of the discussed simplified humidification methods with the extensive method of former investigations within the uncertainty limits mentioned above. Thereby, the calibration laboratories are able to determine humidity coefficients of their transducers at low costs in order to complement the corresponding uncertainty

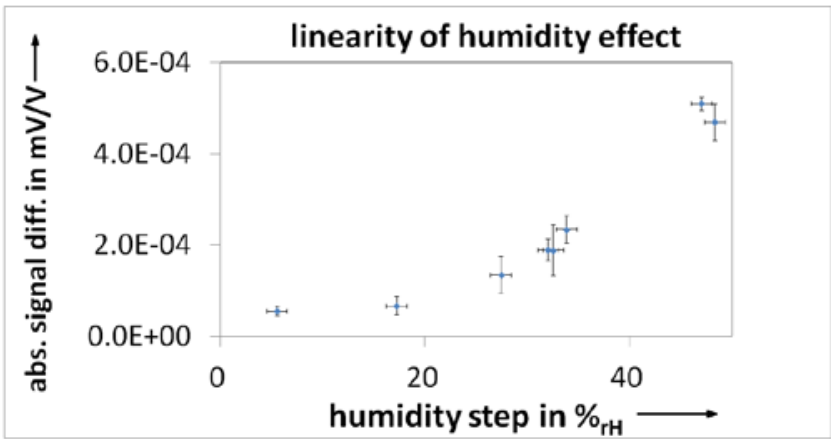

Figure 12. Data of Figure 11, calculated as absolute differences of sensitivities due to humidity steps showing a nonlinear increase with the amount of the humidity step. The error bars are calculated from the uncertainty of the measurements of respectively the humidity steps and the transducer sensitivity (see main text).

budgets. For example, the method of humidification in a local casing is possible with only a small set of cheap and everyday additional equipment as shown in Figure 13 and Figure 6.

A survey of transducers of different types and ranges shows that for state-of-the-art transducers typical absolute values of $\mathrm{h}_{\mathrm{h}}$ are smaller than $10^{-5} / \%_{\mathrm{rH}}$ (Figure 14) and thus compatible to the usual uncertainty budgets in calibration laboratories.

Transducers with unusual designs can vary in their humidity effect both in the time constant and the humidity coefficient. In this manner, transducers could exceed the uncertainty budget of a laboratory which combines small relative uncertainties of $2 \cdot 10^{-4}$ with high limits of relative humidity alteration of more than $20 \% \%_{\mathrm{rH}}$.

One transducer type in the survey is a hermetically sealed transducer, which shows a humidity coefficient below the detection threshold. With the exception of this type, all transducers deliver a negative humidity coefficient.

One transducer aged more than 15 years shows an absolute value of the humidity coefficient higher than $5 \cdot 10^{-5} / \%_{\mathrm{rH}}$. Reasons might be either a design inapplicable in the sense of the humidity effect or a worn out sealing.

The effect of a protective sealing on the strain gauges seems

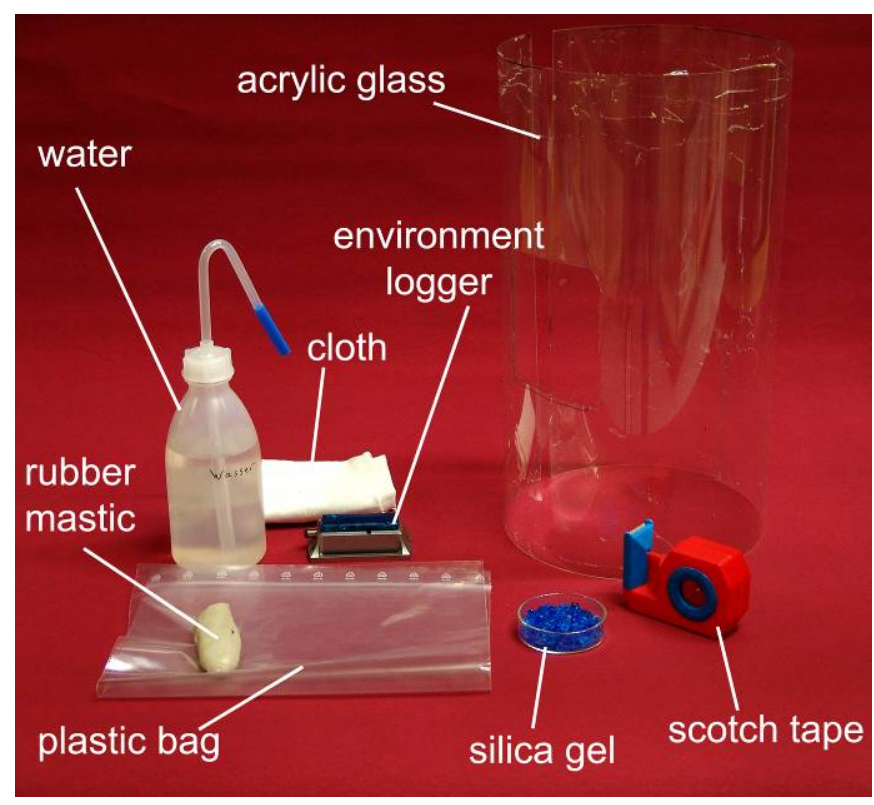

Figure 13: Set of equipment necessary for the in-situ humidification of a torque transducer in a local casing as described in section 3.3. 


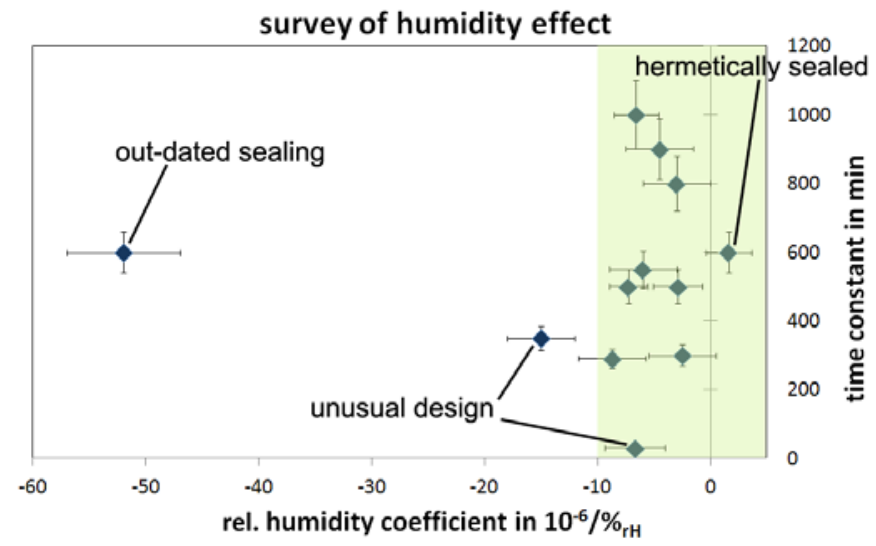

Figure 14. Survey of torque transducers of different types and ranges concerning the relative humidity coefficients and the time constants of the sensitivity response to a humidity step. Transducers within the green area are compatible to the usual needs of calibration laboratories.

to be restricted to the time constant of the sensitivity response to a humidity step. One example in the survey shows a very short time constant of half an hour which points to a weak sealing. Nevertheless, its humidity coefficient is not higher than those of the other transducers with typical time constants of more than 8 hours.

The time constant not only provides data about the quality of the strain gauge sealing but also gives important information about the downtime of a transducer, which has faced humidity conditions far from the laboratory specifications. To this effect, a transfer transducer intended for travelling could benefit from a weak strain gauge sealing, if this would not expand the humidity effect but shorten the time to reconstitute the humidity equilibrium.

In an extended survey (Figure 15) also hand torque tools were tested. Especially the mechanical limiting version of hand torque tools - the click torque wrenches -work without strain gauges. Here the humidity effect comes from the mechanical leverage system whose lubrication grease could be highly hygroscopic. Although these wrenches are meant to work with high uncertainties of some percent, a humidity effect of more

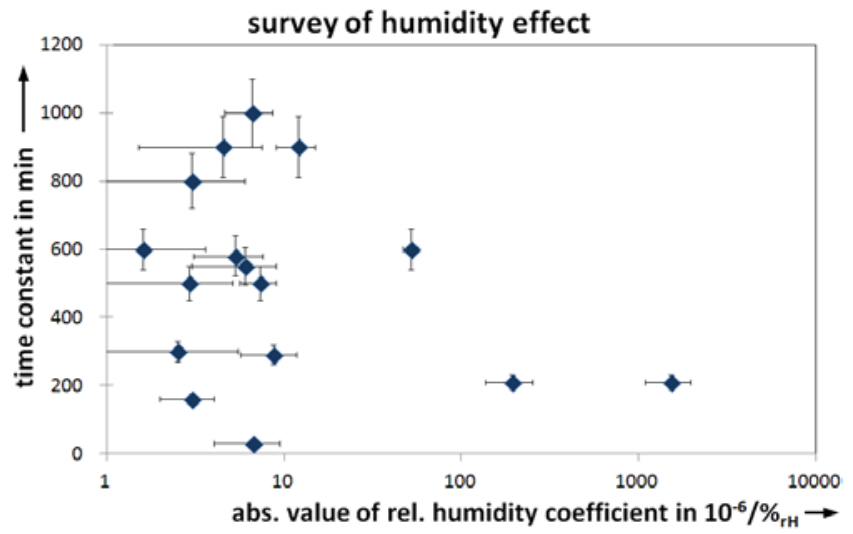

Figure 15. Extended survey of torque sensing instruments including hand torque tools. Some of them, the click torque wrenches, yield humidity effect values of more than $10^{-3} / \% \mathrm{rH}$. This calls for consideration of the humidity effect, even with these apparently tough everyday tools.

than $10^{-3} / \%_{\mathrm{rH}}$ calls for restrictions of the humidity change during their operation.

\section{REFERENCES}

[1] Röske, D., "Key comparisons in the field of torque measurement", 19 $9^{\text {th }}$ International Conference on Force, Mass and Torque (IMEKO TC3): "Theory and Application in Laboratories and Industries", Cairo, 19-23 February 2005, Egypt, on CD-ROM, file name: pdf $\backslash 36$.pdf.

[2] Röske, D.; Mauersberger, D., "On the stability of measuring devices for torque key comparisons", IMEKO XVIII World Congress and IV Brazilian Congress of Metrology, "Metrology for a Sustainable Development", Rio de Janeiro, 17-22 September 2006, Brazil, on CD-ROM, file name: 00181.pdf.

[3] Röske, D., "Final report on the torque key comparison CCM.TK1: Measurand torque: $0 \mathrm{~N} \cdot \mathrm{m}, 500 \mathrm{~N} \cdot \mathrm{m}, 1000 \mathrm{~N} \cdot \mathrm{m}$ ", Metrologia, 46 (2009), Tech. Suppl., 07006, http://dx.doi.org/10.1088/0026-1394/46/1A/07002.

[4] Brüge, A., "Fast Torque Calibrations Using Continuous Procedures", 18 th Conference on Force, Mass and Torque, Celle, Germany, 24-26 September 2002. 